\title{
Real-scale testing of the efficiency of self-healing concrete
}

\author{
K. Van Tittelboom, D. Snoeck, E. Gruyaert, B. Debbaut \& N. De Belie \\ Magnel Laboratory for Concrete Research, Ghent, Belgium
}

\author{
J. Wang \\ Magnel Laboratory for Concrete Research, Ghent, Belgium \\ Laboratory of Microbial Ecology and Technology, Ghent, Belgium
}

\author{
A. De Araújo \\ Magnel Laboratory for Concrete Research, Ghent, Belgium \\ Polymer Chemistry and Biomaterials Group, Ghent, Belgium
}

\begin{abstract}
After several years of research in the Magnel Laboratory (Belgium) to obtain concrete with self-healing properties, the most promising mechanisms were tested on a larger scale. Instead of small mortar samples with self-healing properties, real-scale self-healing concrete beams $(150 \mathrm{~mm} \times 250 \mathrm{~mm} \times 3000 \mathrm{~mm})$ were made and the efficiency of autonomous crack repair was evaluated over time after loading the beams in four-point bending. In addition to a reference beam without self-healing properties, a beam with encapsulated polyurethane and a beam containing superabsorbent polymers were investigated. While for the beam with polyurethane, crack repair is obtained as the healing agent is released as soon as cracks damage the embedded capsules, the superabsorbent polymers absorb water which intrudes into the cracks, immediately blocking the crack through swelling and later on by continued hydration and precipitation of calcite. The efficiency of both self-healing approaches was compared by measuring the reduction in water ingress into the cracks and by measuring the crack width reduction over time.
\end{abstract}

\section{INTRODUCTION}

Concrete is a frequently used construction material because of its low cost, freedom of design, high compressive strength, ... However, while concrete has a high compressive strength, its tensile strength is rather limited. Due to this, concrete often contains cracks within the tensile zones. Although these cracks do not threaten the structural integrity, as reinforcement is provided within these zones to carry the tensile stresses, they do harm the durability of the structure. Via these cracks aggressive agents can enter the matrix and cause concrete degradation e.g. by sulfate attack, chloride ingress, carbonation, ... This may lead to further widening of the cracks and degradation of the reinforcement steel due to corrosion, which can finally result in structural problems. Therefore, it is important that cracks are repaired. However, manual crack repair is expensive and not always feasible because of eventual inaccessibility of cracks.

In 2001, when White et al. (2001) published their paper in Nature about self-healing in polymer based materials, research on the development of self-healing materials gained a real boost. Also attempts were taken to implement self-healing properties inside cementitious materials. Actually, cementitious materials do already have the natural ability to repair damage to a certain extent (Edvardsen 1999). If water enters into concrete cracks and gets into contact with unhydrated cement particles, these can further hydrate and result in crack closure. If both water and carbon dioxide are present, crack closure can be obtained when leaching calcium hydroxide reacts with carbon dioxide to form calcium carbonate crystals. However, as the natural, so called autogenous, crack healing mechanism is limited to small cracks (Reinhardt \& Jooss 2003), many attempts have been made to engineer concrete in order to obtain an improved, autonomous, crack healing efficiency.

Some approaches rather promote the autogenous crack healing ability by limiting the crack width through the addition of polymeric fibers (Li \& Yang 2007) or by the use of shape memory alloys (Jefferson et al. 2009) which return to their original shape, and thus result in crack closure, upon heating. Other approaches promote the autogenous healing ability by providing an extra amount of water through the use of superabsorbent polymers (SAPs) (Lee et al. 2010, Snoeck et al. 2012). If SAPs are embedded in the concrete 
matrix, they absorb water entering via the cracks. The initial swelling action of the SAPs blocks the cracks against further ingress and slow release of the absorbed water, later on, leads to further hydration and calcite precipitation. In other approaches, calcite precipitation is intensified by the addition of micro-organisms to the matrix (Jonkers 2011, Wang 2014). Upon water ingress via the cracks these organisms start to consume available nutrients and precipitate calcium carbonate to close the crack. Next to these self-healing approaches, capsule-based (Dry 2000, Van Tittelboom et al. 2011) and vascular based (Dry 2001, Pareek \& Oohira 2011) mechanisms are reported, in which autonomous crack repair is obtained by release of a, mostly polymer based, healing agent from embedded capsules or from a vascular system. Release of the agent is triggered through breakage of the capsules or the tubes at the moment of crack formation.

In the Magnel Laboratory (Belgium), we started our research on self-healing cementitious materials in 2008. A number of the above mentioned self-healing approaches have been investigated, resulting in diverse self-healing efficiencies. For this study, the most promising approaches were selected to be applied and tested on their efficiency on a larger scale.

\section{MATERIALS}

\subsection{Self-healing mechanisms}

The first self-healing approach which was tested on a larger scale, consisted of the incorporation of encapsulated polyurethane. The polyurethane based healing agent, was a one-component, aircuring agent with a viscosity of $3536 \mathrm{mPas}$ at $25^{\circ} \mathrm{C}$. Although this agent was developed within the framework of another project, it was supposed to meet the requirements in order to obtain efficient self-healing in the large scale concrete beams. This means that the healing agent should remain fluid until capsule breakage, although it should be very reactive to harden as soon as the capsules break and the agent, flowing into the cracks, comes into contact with moisture from the surrounding matrix. In addition, the viscosity of the agent should not be too high, so it is easily released from the capsules and it easily flows into the crack, while at the other hand the viscosity should not be too low, to prevent complete absorption of the healing agent by the surrounding matrix, which would lead to an empty crack. The healing agent was encapsulated by tubular glass capsules with an inner diameter of $3 \mathrm{~mm}$, an outer diameter of $3.35 \mathrm{~mm}$ and a length of about $50 \mathrm{~mm}$. To fill these tubes with the healing agent, first, one end of the tubes was sealed by means of methylmethacrylate (MMA) glue. Then the polyurethane was injected into the tubes by means of a syringe with a needle after which the second end was sealed again with MMA. These brittle capsules were embedded inside the concrete matrix to obtain self-healing properties. As soon as cracks appear in the hardened concrete matrix, the brittle glass capsules crossed by one of these cracks, will break. Due to capillary action, the healing agent will be released from the capsules and will be drawn into the crack. There, the agent will contact moisture inside the pores of the cementitious matrix causing a foaming reaction and hardening of the agent, finally leading to autonomous crack repair.

The second self-healing approach which was ready to be tested on a larger scale, consisted of the use of hydrogels or SAPs. The bulk-polymerized SAPs which were used within this study, were obtained from the company SNF Floerger. They were synthetized from a cross-linked copolymer of acrylamide and acrylate, and had particle sizes less than $600 \mu \mathrm{m}$. This self-healing mechanism is not immediately activated at the moment cracks appear but requires water or moisture ingress into the cracks to become active. When water enters into the cracks, contact with the SAP particles near the crack surface will result in immediate swelling of the particles and thus blockage of the crack. However, over time the particles will release their water content again so the crack blocking effect will be gone but on the other hand the slowly released water will become available to the cementitious matrix and will result in permanent crack closure by further hydration of unhydrated cement particles and calcium carbonate precipitation of leached calcium hydroxide if also carbon dioxide is available within the crack.

\subsection{Concrete beams with(out) incorporated self-healing mechanisms}

One reference beam (code: REF) without self-healing properties was prepared in order to evaluate autogenous healing and to note the additional healing efficiency in the case a self-healing mechanism was embedded. Moulds with dimensions of $150 \mathrm{~mm} \times$ $250 \mathrm{~mm} \times 3000 \mathrm{~mm}$ were used for both the reference beam and the beams with self-healing properties. Preparation of the moulds consisted for the REF beam in placement of the reinforcement bars. As the beams were casted upside down (because later on they would also be loaded in opposite direction) the reinforcement bars were positioned at the upper side of the mould in such a way that the concrete cover on top of the bars would be $20 \mathrm{~mm}$. In total fours bars with a diameter of $10 \mathrm{~mm}$ were equally divided over the width of the mould. 
To cast the real scale concrete beam with embedded polyurethane (code: SHC-PU), in total 350 capsules, filled with polyurethane, were prepared. Positioning of the tubes within the mould was done by means of a network of wires attached through the walls of the mould $10 \mathrm{~mm}$ below the top. Every $40 \mathrm{~mm}$ over the complete length of the beam, except the $160 \mathrm{~mm}$ at the ends, a wire was used to connect the one side of the mould with the other (Fig. 1A and B). Glass tubes were glued onto this network of wires by means of MMA glue. Each time, five capsules were placed with an intermediate distance of $46 \mathrm{~mm}$ and at both capsule ends there was $5 \mathrm{~mm}$ overlap with the wires. The next row was then arranged in an alternating way compared to the previous row. Next to the network of capsules, this beam also contained four reinforcement bars with a diameter of $10 \mathrm{~mm}$. These were positioned underneath the network of capsules at the same position as in the reference beam.

The self-healing approach relying on the swelling and thus blocking effect of SAPs (code: SHC-SAP) did not require additional preparation compared to the reference beams, as the SAPS are added to the concrete upon mixing. It can already be mentioned that the superfluity of additional preparation is an advantage of this self-healing approach compared to the approach with embedded capsules.

After preparation of the moulds, concrete mixes were made. It was decided to cast all beams with self-compacting concrete as vibration of the concrete by means of a needle would be almost impossible for the beam containing the network of wires with capsules. The composition of the concrete mix which was used for each of the beams is shown in Table 1. All ingredients were mixed in a Zyklos 200 litre mixer. The mixing procedure was as follows: sand, gravel, cement, limestone filler (and SAPs) were mixed properly. Subsequently, the needed amount of water was added under continuous mixing. After one minute of mixing, superplasticizer (Glenium 51) was added steadily while mixing persisted. The moulds of the beams were covered with a plastic foil after casting of

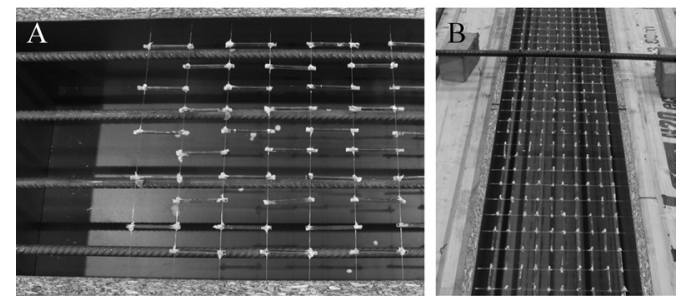

Figure 1. Placement of capsules, filled with polyurethane, on a network of wires which is connected to the sides of the mould (A and B).
Table 1. Composition of the concrete mixes.

\begin{tabular}{llll}
\hline & \multicolumn{3}{l}{ Composition $\left[\mathrm{kg} / \mathrm{m}^{3}\right] /\left[1 / \mathrm{m}^{3}\right]$} \\
\cline { 2 - 4 } Components & REF & SHC-PU & SHC-SAP \\
\hline Sand 0/5 & 853 & 853 & 853 \\
Gravel 2/8 & 370 & 370 & 370 \\
Gravel 8/16 & 328 & 328 & 328 \\
CEM I 52.5 N & 300 & 300 & 300 \\
Limestone filler & 300 & 300 & 300 \\
Water & 165 & 165 & 207 \\
Glenium 51 & 3.33 & 3.00 & 12 \\
SAP & - & - & 3 \\
\hline
\end{tabular}

the beams. The moulds of the beams were removed after six days, then the beams were further stored at room temperature until the time of testing.

\section{METHODS}

\subsection{Crack formation}

In order to create multiple cracks, the beams were loaded in four-point bending. To facilitate performance of the water ingress measurements (see 3.3.1), the beams were loaded in upward direction (Fig. 2). The distance between both loading points amounted to $1000 \mathrm{~mm}$. The metal rollers, used to exert the line loads, were placed symmetrically with regard to the middle of the beam. The rollers of the supports were positioned at the ends of the beam. For one of both supports the displacement was fixed in all directions, while the other one was free in x-direction. To ensure that the load was exactly the same in each of both loading points and that the load values increased at the same speed, one jack was used and the force exerted by this jack was transmitted to both rollers by means of a metal beam (Fig. 2). The exerted force was measured with a load cell. Next to the exerted load, the deformation of the beam was recorded during four-point bending. The curvature of the beam was measured by means of five Linear Variable Differential Transformers (LVDT's, Fig. 2). All LVDT's were positioned at the bottom side (compression zone) of the beam. Making use of the coordinate system shown in Figure 2, they were placed at the following x-positions: LVDT1 at $0 \mathrm{~mm}$, LVDT2 at $800 \mathrm{~mm}$, LVDT3 at $1500 \mathrm{~mm}$, LVDT4 at $2200 \mathrm{~mm}$ and LVDT5 at $3000 \mathrm{~mm}$. As the concrete composition and thus the concrete strength varies for the different beams under investigation (REF: $58.4 \pm 0.9 \mathrm{~N} / \mathrm{mm}^{2}$, SHC-PU: $59.2 \pm 0.4 \mathrm{~N} / \mathrm{mm}^{2}$, SHC-SAP: $47.7 \pm 0.5 \mathrm{~N} / \mathrm{mm}^{2}$ ), not the load or the curvature, but the average crack width was used to control the four-point bending 


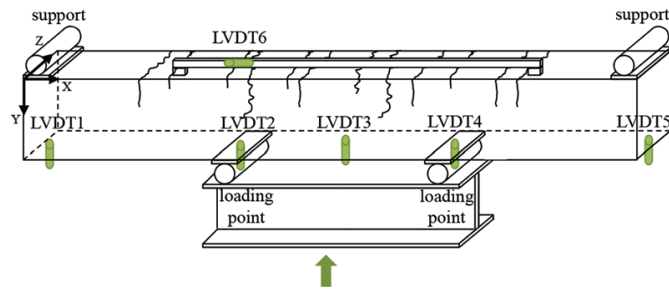

Figure 2. Setup of the four-point bending test with indication of the coordinate system, rods represent the position of the LVDT's, the arrow indicates the load position and direction.

test. Therefore, a measurement frame with a length of $1400 \mathrm{~mm}$ was positioned at the top of the beam (tensile zone), symmetrically with respect to the middle (Fig. 2). The total displacement within the area covered by this measurement frame was measured by an LVDT (LVDT6), which was connected to the frame in horizontal position. The measured displacement is mainly caused by the formation of cracks and further crack opening and partly by strain of the concrete matrix. Supposing that the contribution of concrete elongation is rather limited, the value measured by this LVDT represents the total crack opening within the zone covered by the measurement frame. Division by the total amount of cracks seen within this zone, results in the average crack width.

At the moment of crack formation, beams were loaded in four-point bending until the average crack width amounted to $250 \mu \mathrm{m}$. Note that this value is an overestimation of the real crack width, measured by means of microscopy, as both the strain of the concrete and the total crack width are taken into account when measuring the deformation in the zone covered by the measurement frame. Once this value was reached, the deformation of the beams were fixed and the jack was taken away during the seven weeks period of crack healing.

\subsection{Crack healing}

For the beam with encapsulated polyurethane (SHC-PU), crack formation triggered breakage of the capsules, release of the healing agent and subsequent crack repair when the healing agent came into contact with moisture in the concrete matrix. For the other self-healing approach under investigation (SHC-SAP) contact with water is needed in order to activate the mechanism. As contact with water also induces autogenous crack healing, the natural mechanism of crack healing which is inherent to concrete, it was decided to bring all beams (REF, SHC-PU and SHC-SAP) in contact with water. In this way all beams would exhibit, to some extent, autogenous crack healing and the influence of this effect is filtered out when results are compared. Bringing the beams into contact with water in order to obtain autogenous healing and to activate the self-healing approach with SAP, was done by giving the beams a shower with water four times a day during one minute for a time span of six weeks. Therefore, a plastic tube containing holes, over the complete length of the tube, was positioned in the middle above each of the beams (Fig. 3). At given times, water was pumped through the tubes so it was spraying over the top surface of the beams. As water was subsequently flowing along the sides of the beams, the side surfaces became also wet during each showering cycle. Water which was not absorbed by the cracks or by the concrete matrix was collected, by means of plastic foil, in basins which were positioned underneath the beams.

\subsection{Evaluation of the healing efficiency}

\subsubsection{Crack width reduction}

The initial widths of the cracks in the beams were measured by means of optical microscopy. For each crack, along the whole length of the beam, crack width measurements were performed at 9 positions. 2 measurements at each side of the beam and 5 more measurements of the width at the top surface of the beam. For the beam with encapsulated polyurethane, it was seen immediately that part of the cracks were filled up by polyurethane. During the seven weeks healing period for all of the beams, crack widths became lower due to autogenous (and autonomous) crack healing. Therefore, crack widths were measured a second time after six weeks spraying with water and the crack width reduction over time was calculated.

\subsubsection{Reduction in water ingress}

In order to evaluate the self-healing efficiency, the reduction in water ingress due to crack healing was

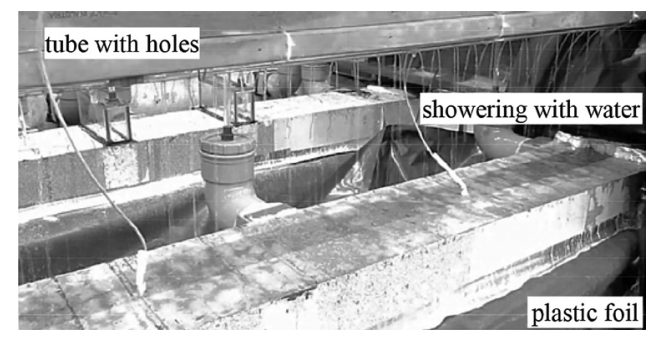

Figure 3. Showering of the concrete beams with water in order to induce autogenous crack healing and trigger the self-healing mechanism based on the use of embedded SAPs. 
measured. After crack formation, plastic basins were glued onto each of the beams, covering 3 zones with a selected crack. The basins were made out of polyvinylchloride and had inside dimensions of $50 \mathrm{~mm} \times 230 \mathrm{~mm}$ (Fig. 4). The height of the basins amounted to $100 \mathrm{~mm}$. At the top of the basins two holes were provided. One was used for water supply and closed during the test by means of a plug. In the second hole, a pipette was positioned with a length of $400 \mathrm{~mm}$ and an inner diameter of $12 \mathrm{~mm}$. This pipette was used for accurate following of the water flow inside the crack during the test. At the bottom of the basin an outlet was provided for fast release of the water after finishing the water ingress test. During the measurement, the sides of the cracks, selected for water ingress, were sealed by means of self-adhesive aluminum butyl tape in order to prevent the water intruding via the crack at the top from leaching out of the crack via the sides of the beam.

Water ingress was calculated using equation 1.

$k=\frac{a T}{A t} \ln \left(\frac{h_{0}}{h_{f}}\right)$

with

$k=$ coefficient reflecting the water ingress $[\mathrm{m} / \mathrm{s}]$

$a=$ cross-sectional area of the pipette $\left[\mathrm{m}^{2}\right]$

$A=$ contact area of water with beam $\left[\mathrm{m}^{2}\right]$

$T=$ height of beam [m]

$t=$ time [s]

$h_{0}$ and $h_{f}=$ initial and final water head (sum of water height in basin and pipette) [m].

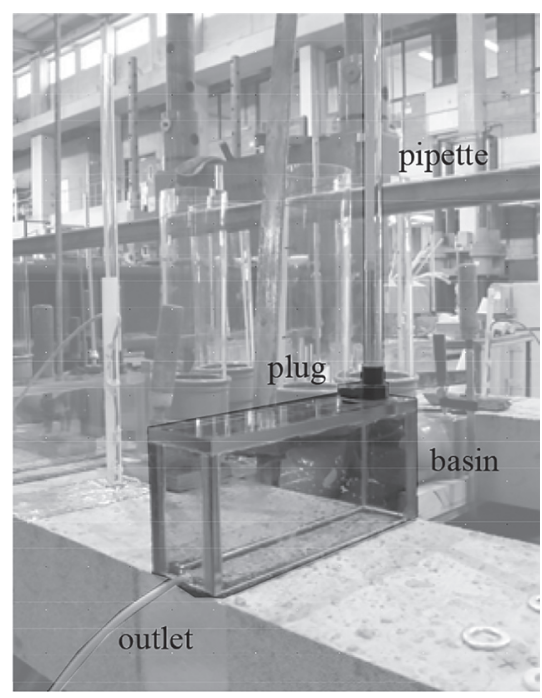

Figure 4. Setup used to measure the water ingress into the cracks.
Water ingress measurements were performed immediately after crack formation and were repeated after crack healing.

\section{RESULTS}

\subsection{Multiple crack formation}

The aim was to investigate whether an improved crack healing efficiency was obtained for the beams with the implemented self-healing approaches (SHC-PU and SHC-SAP) compared to the reference beam (REF). As the crack healing efficiency, particularly the contribution of autogenous healing, depends on the crack width, we aimed for a similar crack width for all of the beams under investigation. Therefore, the beams were not loaded until a predefined load was reached, but they were loaded until an equal average crack width of $250 \mu \mathrm{m}$ was reached. The SAP beam had a lower strength as the SAP particles act as flaws, thus stress initiators (Yao et al. 2011). In Table 2 it can be seen that the number of cracks within the zone covered by the measurement frame (Figure 2) differed for each of the beams, being 20, 22 and 24 for the REF, SHC-PU and SHC-SAP beam, respectively. Due to this, the loading and thus also the elongation of the beams within the middle zone was different. For the REF beam and the SHC-PU beam, the elongation in the middle zone is represented as the sum of two values as the loading was performed in two stages. However, for each of the beams the average crack width (obtained by dividing the total elongation in the middle zone by the number of cracks in the middle zone) amounted to approximately $250 \mu \mathrm{m}$.

In Figure 5 the vertical displacement measured by the LVDT's positioned along the length of the beams is given. For the REF beam and the SHC-PU beam, the displacement is shown as the sum of two bars as these displacements were obtained in two separate loading stages. It can be clearly seen that for LVDT1 and LVDT5, which were placed close to the supports, the measured displacement was very little, as expected. For LVDT2 and LVDT4, placed symmetrically with regard to the middle position of the beam, a similar

Table 2. Multiple crack formation by four-point-bending.

\begin{tabular}{llll}
\hline & $\begin{array}{l}\text { \# cracks } \\
\text { in middle } \\
\text { zone }[-]\end{array}$ & $\begin{array}{l}\text { Elongation } \\
\text { of middle } \\
\text { zone }[\mathrm{mm}]\end{array}$ & $\begin{array}{l}\text { Average } \\
\text { crack width } \\
{[\mu \mathrm{m}]}\end{array}$ \\
\hline REF & 20 & $4.01+1.10$ & 256 \\
SHC-PU & 22 & $4.00+1.55$ & 252 \\
SHC-SAP & 24 & 5.94 & 248 \\
\hline
\end{tabular}



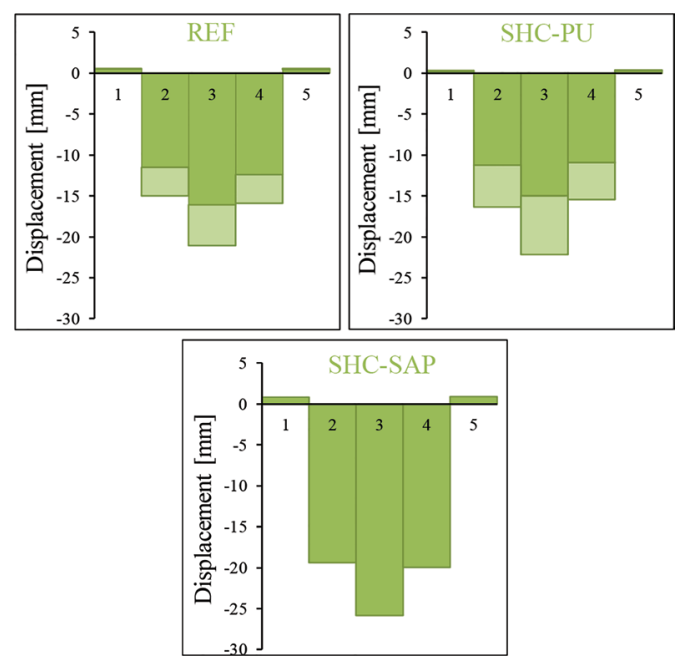

Figure 5. Vertical displacement of the beams at the position of LVDT1, LVDT2, LVDT3, LVDT4 and LVDT5.

negative value for the displacement was measured. As the y-axis is oriented downward (Fig. 2), negative values are obtained for the deformation of the beam loaded in upward direction. The highest negative value was seen in the middle of the beam (LVDT3). As for the SHC-SAP beam more cracks were detected in the middle zone, and thus loading was continued for a longer time, higher absolute values for the displacement were registered. While for the REF beam and the SHC-PU beam the displacement in the middle of the beam amounted to $-20.76 \mathrm{~mm}$ and $-22.91 \mathrm{~mm}$, for the SHC-SAP beam this value amounted to $-25.84 \mathrm{~mm}$.

\subsection{Visualization of crack closure by microscopy}

Due to cyclic exposure of the cracks to water for a period of six weeks, cracks showed some autogenous healing. In Figure 6A it can be seen that for the REF beam some crack healing was noticed, however, this remained limited to the smaller cracks. White precipitates, connected to the crack faces, can be seen. For narrower cracks this led to complete crack healing, however, for larger crack widths we noticed that for the REF beam no complete crack healing could be obtained. This confirms that the natural, autogenous, crack healing mechanism is limited to healing of smaller cracks.

For the beam with encapsulated polyurethane (SHC-PU), the highest portion of crack healing occurred at the moment of crack formation and capsule rupture. However, due to the six weeks spraying with water some additional autogenous healing closed the cracks. This is shown
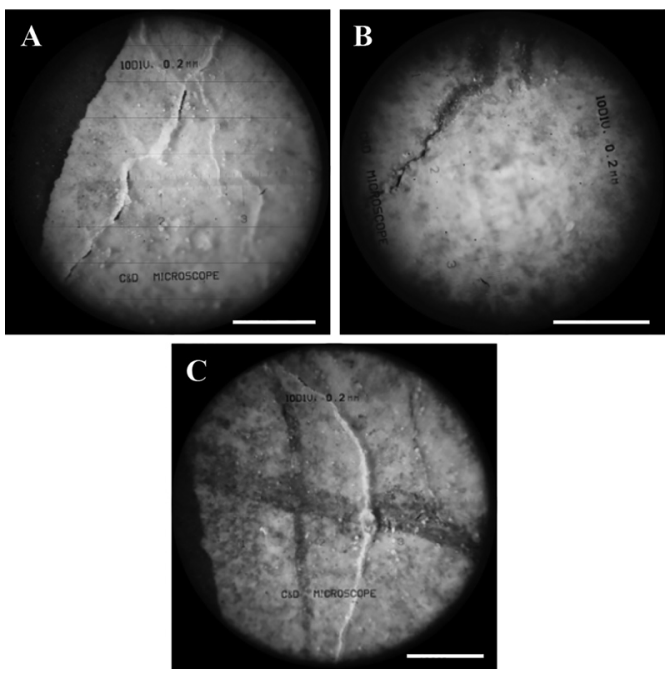

Figure 6. Micrograph of a healed crack inside the reference beam (A), the beams with encapsulated polyurethane (B) and the beams with superabsorbent polymers $(\mathrm{C})$. The white line in the pictures represents a distance of $1 \mathrm{~mm}$.

in Figure 6B. While the upper part of the crack is filled with polyurethane healing agent, it can be seen that the lower part is partly filled with calcium carbonate crystals.

In the beam with superabsorbent polymers improved crack healing was noticed. This can be clearly seen in Figure 6C. In this case more complete crack healing was obtained and also larger cracks could be more completely filled.

\subsection{Crack closing ratio}

The crack closing ratio was calculated as the difference in crack width before and after healing divided by the original crack width. This evaluation procedure only takes into account the amount of healing obtained during the six weeks showering period and not the extent of immediate healing of the beam with encapsulated polyurethane (SHC-PU). Cracks were divided into different categories, based on the original crack width, and crack closing ratios were calculated for each category of each test series.

What immediately can be seen from Figure 7 is that smaller cracks are more likely to be healed compared to wider cracks. For crack widths within the range of 0 to $50 \mu \mathrm{m}$, healing ratios within the range of 40 to $80 \%$ were obtained, while for the cracks within the largest crack width range (200 to $250 \mu \mathrm{m}$ ), healing ratios only amounted to 10 to $30 \%$. A gradual decrease of the healing ratio for 


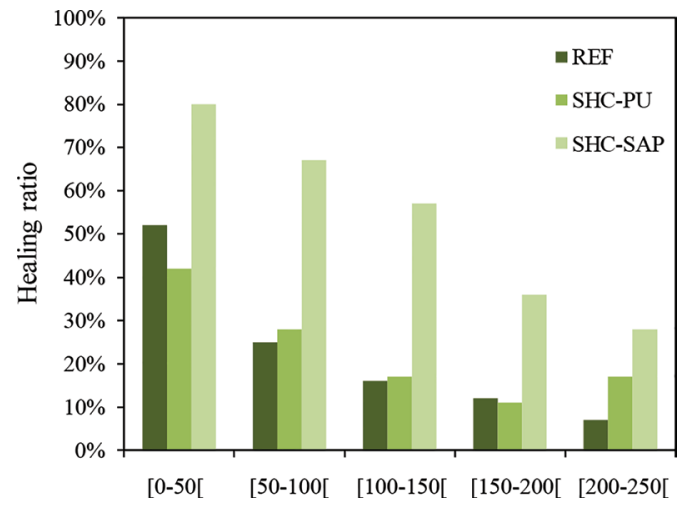

Figure 7. Crack healing ratio obtained within each crack range and for each of the test series under investigation.

each test series can be seen with increasing crack width range. A second very clear finding is that the healing ratio for the beam with embedded SAP particles (SHC-SAP) is considerably higher compared to the healing ratios which are obtained for the two other test series under investigation (REF and SHC-PU, having almost the same healing ratios for each range).

This finding clearly indicates that the addition of SAPs to the concrete matrix promotes crack healing when the crack faces are exposed to water. Due to the addition of SAP, a healing ratio of $80 \%$ was obtained for cracks within the smallest crack width range. But also for the largest range, about $30 \%$ healing was obtained when SAPs were added to the matrix. Although no improved healing ratio is noticed when the SHC-PU beam is compared with the REF beam, this does not mean that healing was inefficient for this series. The healing efficiency of the SHC-PU beams is just not reflected by this experiment as at the time of the initial crack width measurement, autonomous healing already occurred for the SHC-PU beam. This experiment only shows the amount of additional autogenous crack healing which occurred during the seven weeks healing period with six weeks showering.

\subsection{Decrease in water ingress}

As can be seen from Figure 8, before crack healing, the water ingress into the cracks of the beam with embedded SAPs was clearly higher compared to the ingress into the other series (REF and SHC-PU). This is due to the fact that the SAP particles within the matrix of this beam attract an additional amount of water. However, this will result in a beneficial effect later on, as the water, absorbed by the SAPs, will be released to the surrounding cementitious matrix and result in further hydration and

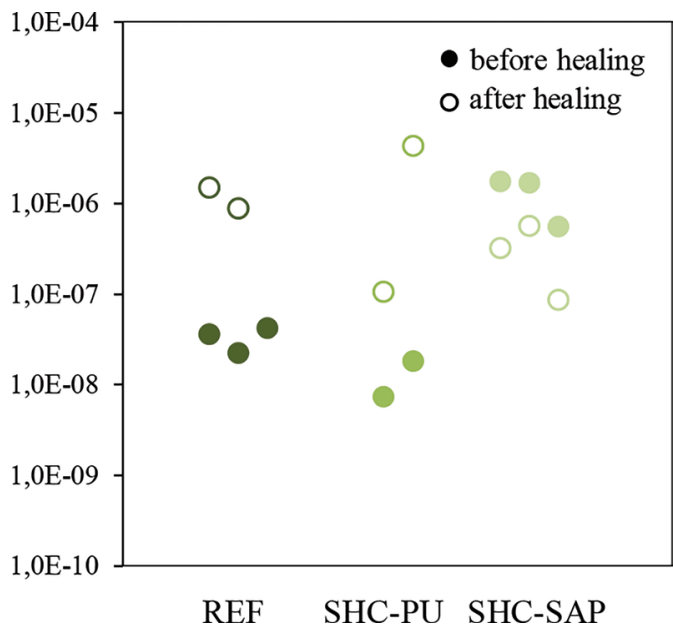

Figure 8. Water ingress into three (some results are missing) selected cracks for each test series measured before and after the healing period.

calcium carbonate precipitation. When these newly formed crystals are precipitated inside the cracks this results in an increased autogenous crack healing efficiency. This improved healing efficiency is partly represented by the results shown in Figure 8 . While for the REF beam and the beam with encapsulated polyurethane (SHC-PU) higher water ingress values were obtained after healing, the SHC-SAP beam showed lower water ingress. We believe this should be attributed to healing of the cracks as for the SAP series crack closure was also shown from the microscopic analysis. The fact that higher water ingress was measured for the two other test series (REF and SHC-PU) is in contradiction with our expectations. However, we believe that this finding is due to the fact that the saturation state of the beams at the moment of the water ingress measurements was different before and after healing. Moreover, these water ingress measurements were very difficult to perform as for some of the selected cracks water was not only intruding in the concrete matrix via the crack but also leaked out of neighboring cracks. This makes it very difficult to draw sound conclusions from this test.

\section{CONCLUSIONS}

From this study it can be concluded that both selfhealing approaches under investigation have some potential to be applied on real scale. However, the use of encapsulated polyurethane requires much more preparation to fill the tubes and position them in the moulds compared to the addition of 
SAPs. Therefore, current research focusses on the development of encapsulation materials with properties evolving over time. So at first they should be flexible and survive concrete mixing while later on the capsules should become very brittle to break at the moment of crack formation. Use of this type of capsules would strongly decrease the time needed for preparation of concrete elements containing encapsulated healing agent. This is subject to further research.

With regard to healing, the approach making use of embedded SAP seems to result in the highest healing efficiency. Crack closure was obviously enhanced through the addition of SAPs to the concrete matrix. This was less clear for the beams with embedded polyurethane, however, the healing efficiency of this approach was not reflected by the crack width measurements. While it was hard to prove a good healing efficiency from the water ingress measurements, after crack formation release of polyurethane from the capsules inside the cracks was clearly noticed.

\section{ACKNOWLEDGEMENTS}

This research under the program SHE (Engineered Self-Healing materials) (project SECEMIN: Selfhealing cementitious and mineral building materials) was funded by SIM (Strategic Initiative Materials in Flanders). The authors would like to thank this foundation for their financial support.

\section{REFERENCES}

Dry C. (2000). Three designs for the internal release of sealants, adhesives, and waterproofing chemicals into concrete to reduce permeability. Cement and Concrete Research 30 (12), pp. 1969-1977.

Dry C. (2001).Design of self-growing, self-sensing, and self-repairing materials for engineering applications. Paper presented at the Smart Materials Conference, pp. 23-29.
Edvardsen C. (1999). Water permeability and autogenous healing of cracks in concrete. ACI Materials Journal 96 (4), pp 448-454.

Jefferson A., Joseph C., Lark R.J., Isaacs B., Dunn S., Weager B. (2010). A new system for crack closure of cementitious materials using shrinkable polymers. Cement and Concrete Research 40 (5), pp. 795-801.

Jonkers H.M. (2011). Bacteria-based self-healing concrete. Heron $56(1 / 2)$, pp. 1-12.

Lee H.X.D., Wong H.S., Buenfeld N.R. (2010). Potential of superabsorbent polymer for self-sealing cracks in concrete. Advances in Applied Ceramics 109 (5), pp. 296-302.

Li V.C., Yang E.H. (2007). Self healing in concrete materials. In: van der Zwaag S (ed) Self healing materials: An alternative approach to 20 centuries of materials science, Springer, pp. 161-193.

Pareek S., Oohira A. (2011). A fundamental study on regain of flexural strength of mortars by using a selfrepair network system. Paper presented at the $3 \mathrm{rd}$ International Conference on Self Healing Materials, Bath, UK, 27-29 June 2011, pp. 46-47.

Reinhardt H-W., Jooss M. (2003). Permeability and selfhealing of cracked concrete as a function of temperature and crack width. Cement and Concrete Research 33 (7), pp. 981-985.

Snoeck D., Van Tittelboom K., Steuperaert S., Dubruel P., De Belie N. (2014). Self-healing cementitious materials by the combination of microfibres and superabsorbent polymers. Journal of Intelligent Material Systems and Structures 25(1), pp. 13-24.

Van Tittelboom K., De Belie N., Van Loo D., Jacobs P. (2011). Self-healing efficiency of cementitious materials containing tubular capsules filled with healing agent. Cement and Concrete Composites 33(4), pp. 497-505.

Wang J., Soens H., Verstraete W., De Belie N. (2014). Self-healing concrete by use of microencapsulated bacterial spores. Cement and Concrete Research 56, pp. 139-152.

WhiteS.R., Sottos N.R., Moore J., Geubelle P., Kessler M., Brown E., Suresh S., Viswanathan S. (2001). Autonomic healing of polymer composites. Nature 409, pp. 794-797.

Yao Y., Zhu Y., Yang Y. (2011). Incorporation of SAP particles as controlling pre-existing flaws to improve the performance of ECC. Construction and Building Materials 28(1) pp. 139-145. 